\title{
Efficient QR-Based Multi-Mode Precoding for Limited Feedback Multi-User MIMO Systems
}

\author{
Chien-Hung Pan • Ta-Sung Lee
}

Published online: 6 August 2013

(C) The Author(s) 2013. This article is published with open access at Springerlink.com

\begin{abstract}
This paper proposes QR-based criteria for efficient selection of multi-mode precoders in multi-user multiple-input multiple-output (MU-MIMO) precoded systems with a limited feedback. In particular, a MU-MIMO QR based precoding scheme is developed which takes advantage of the overall precoder design among users in conjunction with $\mathrm{QR}$ detection. A selection criterion is developed to identify the precoder that yields the maximum free distance. Efficient mode selection and tree search strategies are adopted to reduce the complexity associated with the identification of the optimum precoder. To further improve the detection performance in correlated channels, an efficient QR-based MMSE V-BLAST detector is proposed. Computer simulations confirm that the proposed QR-based precoding scheme attains the performance of existing schemes with a significantly lower complexity level.
\end{abstract}

Keywords QR decomposition · Multi-mode precoding · Multiple-input multiple-output . Limited feedback system

\section{Introduction}

The recent increase in demand for higher spectral efficiency prompts the development of uplink single-user MIMO (SU-MIMO) system in the LTE-advanced standard $[1,2]$. The SU-MIMO system was proposed using a structured precoding codebook and the limited feedback technique [3]. The limited feedback associated with the precoder index comprises fewer bits because codebooks are stored in both the transmitter and receiver and the receiver sends the index of the selected precoder to the transmitter via a limited feedback channel $[4,5]$. For the limited feedback in the SU-MIMO system, effective quantization schemes for channel status information (CSI) feedback were developed

C.-H. Pan $(\varangle) \cdot$ T.-S. Lee

Department of Electrical Engineering, National

Chiao Tung University, Hsinchu, Taiwan

e-mail: andypan.taiwan@gmail.com 
$[4,6]$. Various selection criteria with exhaustive search were proposed to identify the precoder and mode to optimize transmission performance [7-9]. An extension of the SU-MIMO system to the multi-user MIMO (MU-MIMO) system was intended to provide space-division multiple access [10,11]. However, designing multi-mode precoding for MU-MIMO to improve detection performance is even more challenging due to non-cooperative users (i.e., mobile stations) and the complex computations as the number of users grows [12]. Overcoming these difficulties is the motivation for this paper.

In the proposed multi-mode precoding, the receiver (i.e., the base station) collects the CSI associated with respective users and designs the overall precoder. Users and receivers are equipped with multiple transmit and receive antennas and each user can transmit either a single symbol stream or multiple streams to the receiver. According to the condition of the overall channel, each user adopts the precoder determined by the receiver to precode its own signal. Specifically, the precoders obtained from the respective user's codebook are combined to form the overall precoder. We here propose a QR-based criterion for the selection of the overall precoder to minimize the probability of detection error [7]. This is accomplished by maximizing the minimum absolute value of diagonal entries in the upper triangular matrix of the QR-decomposition (QRD) associated with the effective channel. The effective channel matrix is the product of the overall channel matrix and the overall precoder matrix, which varies according to the overall precoder. The detection performance is optimized by selecting the precoder according to the largest minimum absolute value of the diagonal entries. Thus, the overall precoder produces an effective channel matrix yielding a near equal-diagonal QRD [14,15], leading to the maximum free distance or the minimum rate of error detection.

For large codebooks and/or a large number of users, the design of the overall precoder inevitably requires complex computations since the users' precoders are obtained through an exhaustive search over all possible combinations. To reduce the search complexity, efficient mode selection (EMS) and partial precoder search strategies are developed using the SVD of the original sub-channel matrix for each user. EMS in QR-based selection criterion is also capable of yielding the maximum free distance to identify the precoder. The partial precoder search is performed by converting the precoders in a codebook into a binary tree $[16,17]$, which reduces the number of candidate precoders using the concept of short distance within a limit region. To further improve the detection performance in correlated channels, efficient QR-based MMSE V-BLAST detection [18,19] is proposed, which employs a low-complexity scheme for computing the nulling vectors. Simulation results show that the proposed EMS and partial precoder search schemes are capable of achieving nearly the optimal performance with a very low level of complexity.

This paper is organized as follows. In Sect. 2, we describe the uplink MU-MIMO system for multi-mode precoding. In Sect. 3, we present the QR-based criteria for low-complexity selection in multi-mode precoding and the column ordering scheme. In Sect. 4, the performance of the proposed QR-based precoding scheme is analyzed. In Sect. 5, we conduct computer simulations to confirm the effectiveness of the proposed algorithms. Section 6 concludes the paper.

Notation: Matrices and vectors are denoted by upper and lower case boldface letters respectively. $\mathbf{I}_{M}$ is the $M \times M$ identity matrix. For vector $\mathbf{x}, x_{i}$ is its $i$ th entry. Superscript $(\cdot)^{H},(\cdot)^{-1}$ and $(\cdot)^{\dagger}$ represent the Hermitian transpose, inverse and pesudoinverse operations, respectively. $|\cdot|$ denotes the absolute value operator, $\|\cdot\|_{F}$ and $\|\cdot\|_{2}$ represent Frobenius norm and 2-norm of a matrix, respectively, $\|\cdot\|$ denotes the $L_{2}$-norm of a vector and blkdiag(·) denotes the block diagonal operation. 


\section{System Model of Multi-Mode Precoding}

In this section, QR-based multi-mode precoding for the uplink MU-MIMO system is described. We first briefly review system model for the multi-user multi-mode precoding. Then, the QRD for MU-MIMO precoding under non-cooperative users is described. Finally, the QR-based multi-mode precoding strategy is investigated to calculate the minimum distance to optimize the MU-MIMO precoding system. The proposed schemes are illustrated in 2.1. Multi-user multi-mode precoding, 2.2. QR detection in precoded system, and 2.3. QR-based multi-mode precoding scheme.

\subsection{Multi-User Multi-Mode Precoding}

As illustrated in Fig. 1, we consider multi-mode precoding with $M_{t}=N_{1}+N_{2} \cdots+N_{Q}$ transmit antennas, where $N_{q}$ is the number of transmit antennas at the $q$ th user, $1 \leq q \leq Q$, and $M_{r}$ receive antennas in a limited feedback uplink MU-MIMO system [20]. The overall $W=W_{1}+W_{2} \cdots+W_{Q}$ transmitted bits, with $W_{q}$ being the number of transmitted bits of the $q$ th user, are mapped to $B=M_{1}+M_{2} \cdots+M_{Q}$ different data streams, with $M_{q}$ being the number of data streams (or mode) of the $q$ th user, $1 \leq M_{q} \leq N_{q}$. Without inter-user cooperation, the signal model can be described as

$$
\mathbf{y}=\mathbf{H F}_{M} \mathbf{x}+\mathbf{v},
$$

where $\mathbf{y} \in C^{M r \times 1}$ is the received signal vector, $\mathbf{x} \in C^{B \times 1}$ is the transmitted symbol vector with the correlation matrix $E_{\mathbf{x}}\left[\mathbf{x x}^{H}\right]=\left(\varepsilon_{\mathbf{x}} / B\right) \mathbf{I}_{B}, \mathbf{H} \in C^{M r \times M t}$ is the MIMO channel matrix with complex Gaussian entries which can be divided into $Q$ subchannels as $\mathbf{H}=$ $\left[\mathbf{H}_{1} \mathbf{H}_{2} \ldots \mathbf{H}_{Q}\right]$ and then the matrix $\mathbf{F}_{M}=\left[\mathbf{F}_{M 1} \mathbf{F}_{M 2} \ldots \mathbf{F}_{M Q}\right] \in C^{M t \times B}$ with $\mathbf{F}_{M}^{H} \mathbf{F}_{M}=\mathbf{I}_{B}$ is the precoder matrix with $E_{\mathbf{X}}\left[\left(\mathbf{F}_{M} \mathbf{x}\right)^{H} \mathbf{F}_{M} \mathbf{x}\right] \leq \varepsilon_{\mathbf{X}}$ regardless of the modulation scheme or the value of $B, M=\left(M_{1}, M_{2}, \ldots, M_{Q}\right) \in \mathcal{M}$ represents a specific combination of user modes, $\mathbf{v} \in C^{M r \times 1}$ is the noise vector with i.i.d. complex Gaussian entries and power $\sigma_{v}^{2}$ and $E(\cdot)$ is the expectation operator.

When $\mathbf{H}$ changes, the overall precoder matrix $\mathbf{F}_{M}=\operatorname{blkdiag}\left(\mathbf{F}_{M 1}, \mathbf{F}_{M 2}, \ldots, \mathbf{F}_{M Q}\right)$ is adapted using the condition of the current channel based on the parameters received from a feedback channel, as depicted in Fig. 1. The precoder matrix $\mathbf{F}_{M q}$ of the $\left(M_{q}\right)$ th transmission mode of the $q$ th user is selected from the $N_{M q}$ different $M_{t}^{\times} M_{q}$ precoder matrices denoted as

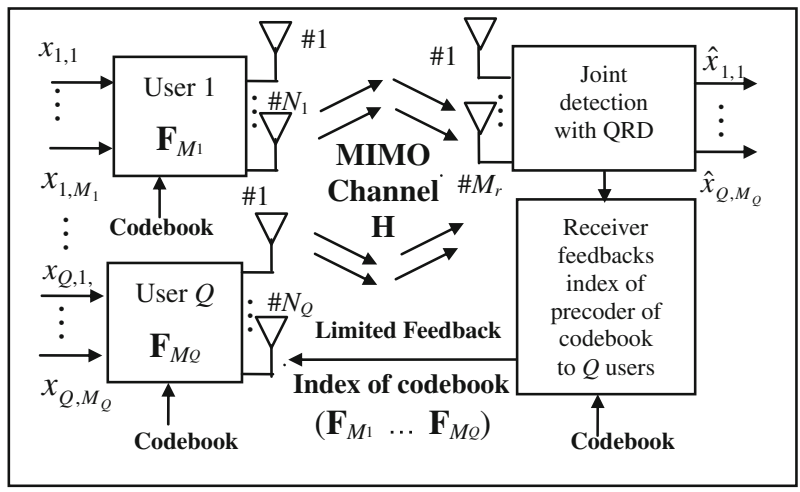

Fig. 1 A precoded uplink MU-MIMO system with limited feedback in which the receiver transmits the index of precoder of codebook to users via a limited feedback link 


$$
\mathcal{F}_{M_{q}}=\left\{\mathbf{F}_{M_{q}, 1}, \mathbf{F}_{M_{q}, 2}, \ldots, \mathbf{F}_{M_{q}, N_{M q}}\right\}
$$

where $M_{q} \in \mathcal{M}_{q}$ and $\mathcal{M}_{q}$ is a set of supported modes for the $q$ th user. For example, if $W=M_{t}=M_{r}=4$ and $Q=N_{q}=W_{q}=2$, the BF (beamforming) mode with 4-QAM adopted in each data stream and the SM (spatial multiplexing) mode with BPSK adopted in each data stream are supported for the $q$ th user with the mode set $\mathcal{M}_{q}=\{1,2\}$. Thus, the overall supported transmission modes for the $Q=2$ users are given by $M=\left(M_{1}, M_{2}\right) \in$ $\{(\mathrm{BF}, \mathrm{BF}),(\mathrm{BF}, \mathrm{SM}),(\mathrm{SM}, \mathrm{BF}),(\mathrm{SM}, \mathrm{SM})\}$ or $\mathcal{M}=\{(1,1),(1,2),(2,1),(2,2)\}$.

\subsection{QR Detection in Precoded System}

We first consider the QR detection for MU-MIMO precoding systems. In QR-based detection [13], the QRD of the effective channel $\mathbf{H F}_{M}$ is given by

$$
\mathbf{H F}_{M}=\left[\begin{array}{ll}
\mathbf{Q}_{1} & \mathbf{Q}_{2}
\end{array}\right]\left[\begin{array}{l}
\mathbf{R} \\
\mathbf{0}
\end{array}\right]=\mathbf{Q R},
$$

where $\mathbf{Q}=\left[\mathbf{Q}_{1} \in C^{M r \times B} \mathbf{Q}_{2} \in C^{M r \times(M r-B)}\right]$ is an $M_{r} \times M_{r}$ unitary matrix and $\mathbf{R}$ is an $B \times B$ upper triangular matrix. The QR-based detection is performed as follows:

$$
\tilde{\mathbf{y}}=\left[\begin{array}{l}
\tilde{y}_{1} \\
\tilde{y}_{2} \\
\vdots \\
\tilde{y}_{M_{r}}
\end{array}\right]=\mathbf{Q}^{H} \mathbf{y}=\underbrace{\left[\begin{array}{cccc}
r_{1,1} & r_{1,2} & \cdots & r_{1, B} \\
0 & r_{2,2} & \cdots & r_{2, B} \\
\vdots & \vdots & \cdots & \vdots \\
0 & \cdots & 0 & r_{B, B}
\end{array}\right]}_{\mathbf{R}} \mathbf{x}+\tilde{\mathbf{v}}=\mathbf{R} \mathbf{x}+\tilde{\mathbf{v}},
$$

where $\mathbf{R}=\mathbf{Q}^{H} \mathbf{H}, \tilde{\mathbf{v}}=\mathbf{Q}^{H} \mathbf{v}$, and $r_{i, j}, 1 \leq i, j \leq B$, is the $(i, j)$ th entry of $\mathbf{R}$. The $i$ th entry of the modified received signals is detected as

$$
\hat{y}_{i}=\tilde{y}_{i}-\sum_{j=i+1}^{B} r_{i, j} \hat{x}_{j}=r_{i, i} x_{i}+\sum_{j=i+1}^{B} r_{i, j}\left(x_{j}-\hat{x}_{j}\right)+\tilde{v}_{i},
$$

where $\hat{x}_{j}$ is the $j$ th entry of the detected symbol vector. Assuming that there is no error in the previous symbol detection [13], we obtain

$$
\hat{x}_{i}=\operatorname{Decision}\left(\frac{\hat{y}_{i}}{r_{i, i}}\right) \text {, where } \hat{y}_{i}=r_{i, i} x_{i}+\tilde{v}_{i} .
$$

\subsection{QR-Based Multi-Mode Precoding}

In this subsection, along the line of $\mathrm{QR}$ detection, a low-complexity QR-based precoder selection criterion is developed to select the precoder and mode to implement the MU-MIMO precoding to optimize the limited feedback system. First, the minimum distance of the constellation $\chi^{M q}$ at the $q$ th user is defined by

$$
d_{\min }\left(M_{q}, W_{q}\right)=\min _{\mathbf{s}_{q}, \mathbf{c}_{q} \in \chi^{M_{q}}, \mathbf{s}_{q} \neq \mathbf{c}_{q}}\left|\mathbf{s}_{q}-\mathbf{c}_{q}\right|,
$$

where $\mathbf{s}_{q}$ and $\mathbf{c}_{q}$ are two different signal vectors with the modulation of $W_{q} / M_{q}$ bits per substream [4]. The minimum distance in the MU-MIMO precoded system may be untraceable due to that there is no cooperation between users at the transmitter such that different users may adopt different modulations. However, a lower bound can be obtained as shown in the following Lemma. 
Lemma 1 Assuming that there are no cooperation at the transmitter for the MU-MIMO system, the minimum distance of the MU-MIMO system is larger than or equal to the minimum distance of the SU-MIMO system under the condition that both systems have the same number of transmit antennas, receive antennas and total number of transmitted bits per transmission.

Proof Under above conditions, the minimum number of data streams in the MU-MIMO system is $B=Q \geq 1$ which is larger than or equal to the number of a single data stream for the BF transmission in the SU-MIMO system. Thus the proof is complete.

Based on Lemma 1, the minimum distance of the MU-MIMO precoded system can be approximated as

$$
\tilde{d}_{\min }(B, W) \approx \sum_{q=1}^{Q} d_{\min }\left(M_{q}, W_{q}\right) .
$$

With (8), the QR-based selection criterion [7] is proposed to determine the overall precoder to optimize MU-MIMO precoding as follows:

$$
\begin{aligned}
\mathbf{F}_{M} & =\underset{\mathbf{F}^{\prime} \in \mathcal{F}_{M}}{\arg \max } \min _{1 \leq i \leq B}\left|r_{i, i}\left(\mathbf{H F}^{\prime}\right)\right|, \\
\mathbf{F}_{\mathrm{QR}} & =\underset{M \in \mathcal{M}}{\arg \max }\left|r\left(\mathbf{H F}_{M}\right)\right| \cdot \tilde{d}_{\min }(B, W),
\end{aligned}
$$

where $r_{i, i}\left(\mathbf{H} \mathbf{F}^{\prime}\right)$ is the $(i, i)$ th entry of $\mathbf{R}$ in the $\mathrm{QRD}$ of $\mathbf{H} \mathbf{F}^{\prime}, r\left(\mathbf{H F} \mathbf{F}_{M}\right)$ is the optimal diagonal entry associated with mode $M$ obtained in (9) and the overall codebook of the $M$ th mode can be generated by $\mathcal{F}_{M}=\operatorname{blkdiag}\left(\mathcal{F}_{M 1}, \mathcal{F}_{M 2}, \ldots, \mathcal{F}_{M Q}\right)$, where blkdiag( $)$ denotes the block diagonal matrix formed by the matrix entries. Note that the precoder is determined in (9) to maximize the minimum absolute value of diagonal entries of $\mathbf{R}$ in each mode of codebook. The optimal mode in (10), which invokes the largest bound, is selected by computing the minimum distance bound for each mode. The proposed QR-based selection criterion, using the exhaustive search in (9) and (10), is performed on minimizing the probability of detection error. Finally, the receiver sends the indices of the precoders selected from the codebooks to the users via a limited feedback channel.

\section{Efficient QR-Based Multi-Mode Precoding and Detection Schemes}

To lessen the search complexity in this section, alternative efficient schemes based on the QRD are proposed to achieve the multi-mode precoding and detection in a limited feedback MU-MIMO system. With maximizing the free distance to design a good precoder strategy, channel's free distance involved diagonal entries of the R-factor of QRD is firstly developed in EMS to determine the transmission mode. Based on this transmission mode, the treestructured codebook in terms of the singular value vector of channel is building to cluster few precoder candidates to reduce the complexity of QRDs. The proposed schemes are illustrated in 3.1. EMS, 3.2. Efficient precoder selection via tree search algorithm, and 3.3. Efficient QR-based MMSE V-BLAST detection as follows.

\subsection{Efficient Mode Selection}

For a large number of users, (9) and (10) become computationally intensive to perform the exhaustive search by doing total QRDs to find the optimum precoder. We here suggest a lowcomplexity method with EMS to reduce the complexity. This approach performs fewer QRDs 
by exploiting the SVD of the original sub-channel matrix with which each user computes the minimum distance bound for each mode by the following property to choose a suitable reference precoder $\mathbf{E}_{M q}$ :

Property 1 Given the $\operatorname{SVD~} \mathbf{H}_{q}=\mathbf{U}_{q} \mathbf{\Lambda}_{q} \mathbf{V}_{q}^{H}$ with singular values $\lambda_{1} \geq \lambda_{2} \geq \cdots \geq \lambda_{N_{q}}>0$, we have the QRD $\mathbf{H}_{q} \mathbf{V}_{M q}=\mathbf{U}_{q} \boldsymbol{\Lambda}_{M q}$, where $\mathbf{V}_{M q}$ consists of the first $M_{q}$ columns of $\mathbf{V}_{q}^{H}$, and $\boldsymbol{\Lambda}_{M q}$ consists of the first $M_{q}$ columns of $\boldsymbol{\Lambda}_{q}$.

The proof is straightforward and thus omitted for brevity. It follows that a reasonable choice for the reference precoder is $\mathbf{E}_{M q}=\mathbf{V}_{M q}$ for $M_{q}<N_{q}$ because it gives an effective channel matrix with $M_{q}$ largest diagonal entries in its QRD.

For $M_{q}=N_{q}$, the reference precoder is chosen to be $\mathbf{E}_{M q}=\mathbf{P}_{q}$, where $\mathbf{H}_{q}=\mathbf{Q}_{q} \mathbf{R}_{q} \mathbf{P}_{q}^{H}$ is the GMD of $\mathbf{H}_{q}$. Recall that the GMD based precoder provides the optimum performance by exploiting equal QRD $[14,15]$. Based on the selection of the reference precoder $\mathbf{E}_{M q}$, the free distance [14] for the $\left(M_{q}\right)$ th transmission mode at the $q$ th user can be achieved as follows:

Theorem 1 Assuming $\mathbf{H}_{q} \in C^{M r \times N q}$ with singular values $\lambda_{1} \geq \lambda_{2} \geq \cdots \geq \lambda_{N_{q}}>0$, the reference precoder $\mathbf{E}_{M q}$ satisfies

$$
d_{\text {free }}\left(\mathbf{H}_{q} \mathbf{E}_{M_{q}}\right)=\min _{1 \leq i \leq M_{q}}\left|r_{i, i}^{q}\left(\mathbf{H}_{q} \mathbf{E}_{M_{q}}\right)\right| \cdot d_{\min }\left(M_{q}, W_{q}\right),
$$

where $r_{i, i}^{q}\left(\mathbf{H}_{q} \mathbf{E}_{M q}\right)$ is the (i,i)th entry of the diagonal matrix $\boldsymbol{\Lambda}_{M q}$ of $Q R D$ of $\mathbf{H}_{q} \mathbf{E}_{M q}$ as depicted in Property $1, d_{\min }\left(M_{q}, W_{q}\right)$ is the minimum distance in (7) and $d_{\text {free }}\left(\mathbf{H}_{q} \mathbf{E}_{M q}\right)$ is the free distance of $\mathbf{H}_{q} \mathbf{E}_{M q}$. Moreover, the free distance in (11) can be maximized.

\section{Proof See "Appendix 1".}

Using (11), the optimum transmission mode and corresponding precoder that minimizes the error probability can be obtained by

$$
\begin{aligned}
M_{q} & =\underset{M_{q} \in \mathcal{M}_{q}}{\arg \max } \min _{1 \leq i \leq M_{q}}\left|r_{i, i}^{q}\left(\mathbf{H}_{q} \mathbf{E}_{M_{q}}\right)\right| \cdot d_{\min }\left(M_{q}, W_{q}\right), \\
\mathbf{F}_{Q R} & =\underset{\mathbf{F}_{M q} \in \mathcal{F}_{M_{q}}}{\arg \max } \min _{1 \leq i \leq B}\left|r_{i, i}\left(\left[\mathbf{H}_{1} \mathbf{F}_{M_{1}} \cdots \mathbf{H}_{q} \mathbf{F}_{M_{q}} \cdots \mathbf{H}_{Q} \mathbf{F}_{M_{Q}}\right]\right)\right| .
\end{aligned}
$$

In contrast to the exhaustive search in (9)-(10), the transmission mode of each user can be independently determined beforehand enabling the optimal precoder to be identified within the selected mode.

\subsection{Efficient Precoder Selection Via Tree Search Algorithm}

To reduce the search complexity, the modified random vector quantization codebook with binary tree structure is developed in Santipach [16] to select the vector to maximize capacity. Based on this structure, the tree search technique is adopted to avoid the exhaustive QRDs in (9) and (13) in finding the precoder. The precoders of the $M_{q}^{\text {th }}$ codebook converted into a binary search tree exploited by the constellation point, using a separation of real and imaginary, are firstly developed in Table 1 . Partial precoder search can then be conducted using the binary search tree [17] in which (1) the left subtree of a node includes only nodes with numbers smaller than the node's number, (2) the right subtree of a node includes only nodes with numbers larger than or equal to the node's number. 
Table 1 Conversion of codebook into a binary tree

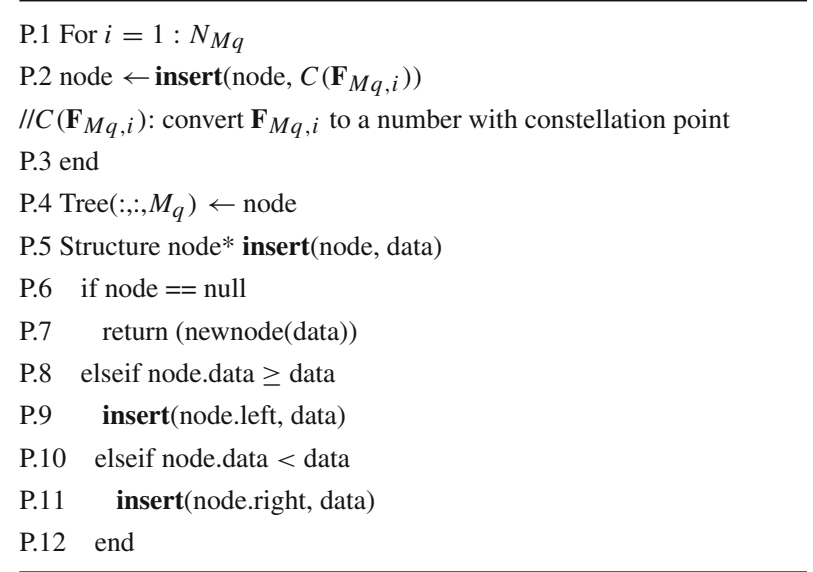

Based on Table 1, the precoder can be further determined by the following optimization problem as

$$
\begin{aligned}
\mathbf{F}_{T, M_{q}}=\arg & \min _{1 \leq j \leq N_{M_{q}}, \mathbf{F}_{M_{q, j}} \in \mathcal{F}_{M_{q}}}\left(\sum_{t=1}^{M_{t}} \sum_{b=1}^{B}\left|\operatorname{Re}\left(\mathbf{F}_{M_{q}, j}(t, b)-\mathbf{E}_{M_{q}}(t, b)\right)\right|+\mid \operatorname{Im}\left(\mathbf{F}_{M_{q}, j}(t, b)\right.\right. \\
& \left.\left.-\mathbf{E}_{M_{q}}(t, b)\right) \mid\right),
\end{aligned}
$$

where $\operatorname{Re}(\cdot)$ and $\operatorname{Im}(\cdot)$ denote the real part and the imaginary part of $(\cdot)$, respectively. To avoid the exhaustive search, the tree search is employed to determine the precoder $\mathbf{F}_{T, M q}$ in (14) with which a partial set of $C_{M q}$ adjacent nodes in the tree of the $M_{q}^{\text {th }}$ codebook are selected $[16,17]$. Candidate precoders of the reduced set $\mathcal{P}_{M_{q}}$ are then selected from the adjacent nodes of $\mathbf{F}_{T, M q}$ as given by

$$
\mathcal{P}_{M_{q}} \leftarrow C_{M_{q}}^{\prime} \text { s adjacent nodes of } \mathbf{F}_{T, M_{q}} \text {. }
$$

Particularly, absolute value and subtraction in (14) to cluster few candidate precoders have less computational complexity than the QRD in (13). Thus, the tree search reduces the complexity of $\left(N_{M 1} \cdot N_{M 2} \cdot \ldots N_{M Q}\right)$ QRDs to $\left(C_{M 1} \cdot C_{M 2} \cdot \ldots C_{M Q}\right)$ QRDs in finding the precoders. With (15), the optimum transmission mode and corresponding precoder can be obtained by

$$
\begin{aligned}
M_{q} & =\underset{M_{q} \in \mathcal{M}_{q}}{\arg \max } \min _{1 \leq i \leq M_{q}}\left|r_{i, i}^{q}\left(\mathbf{H}_{q} \mathbf{E}_{M_{q}}\right)\right| \cdot d_{\min }\left(M_{q}, W_{q}\right), \\
\mathbf{F}_{Q R} & =\underset{\mathbf{F}_{M q} \in \mathcal{P}_{M q}}{\arg \max } \min _{1 \leq i \leq B}\left|r_{i, i}\left(\left[\mathbf{H}_{1} \mathbf{F}_{M_{1}} \cdots \mathbf{H}_{q} \mathbf{F}_{M_{q}} \cdots \mathbf{H}_{Q} \mathbf{F}_{M_{Q}}\right]\right)\right|,
\end{aligned}
$$

which shows that a transmission mode can be firstly determined in (16) and then only the partial set of precoders in $\mathcal{P}_{M q}$ need be computed to find the precoder in (17). The procedure is depicted in Table 2.

\subsection{Efficient QR-Based MMSE V-BLAST Detection}

In MU-MIMO precoding, the total number of data streams is at least $Q$, as mentioned in Lemma 1. In this case, conventional QR-based detection may fail to separate the data streams of different users under correlated channels because of the ill-conditioned channel matrix. In $[18,19]$, QR-based detection methods have been proposed to reduce the complexity of 
Table 2 Proposed tree search based precoding with $Q=2$
S.1 Precoders of each codebook are converted into binary tree

S.2 $\mathbf{E}_{M q}$ is computed by SVD or GMD of $\mathbf{H}_{q}$

S.3 EMS is used to determine the mode for respective users

S.4 $\mathbf{F}_{T, M q} \leftarrow \operatorname{search}\left(\operatorname{Tree}\left(:,:, M_{q}\right), C\left(\mathbf{E}_{M q}\right)\right)$

$/ / C\left(\mathbf{E}_{M q}\right)$ : convert $\mathbf{E}_{M q}$ to a number with constellation point

S.5 Find $C_{M q}$ 's adjacent nodes of $\mathbf{F}_{T, M q}$ to be $\mathcal{P}_{M q}$

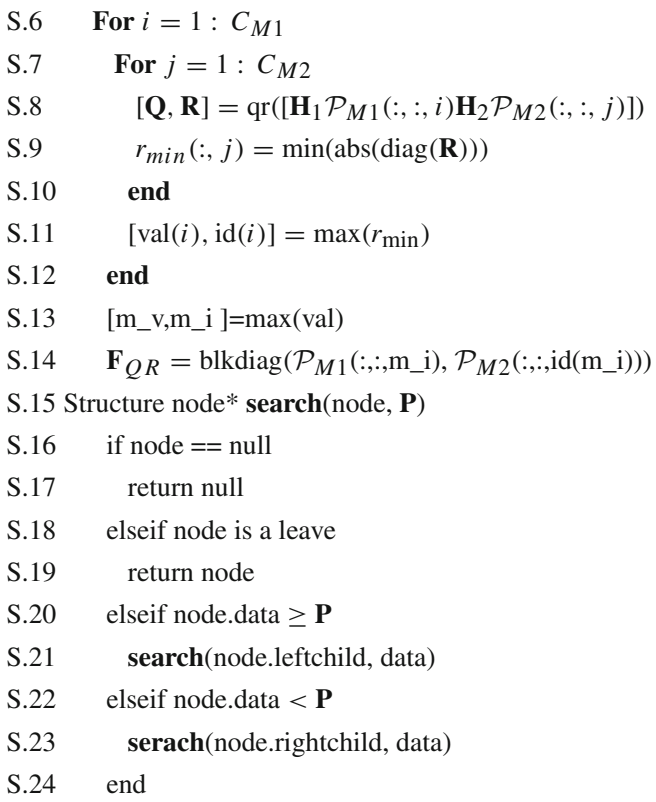

MMSE V-BLAST. These modified MMSE V-BLAST detectors are robust to ill-conditioned channels and are suitable for the MU-MIMO precoded system. On the other hand, detection ordering is essential in order to avoid error propagation. In this subsection, an efficient scheme is introduced to implement QR-based MMSE V-BLAST detection. The proposed scheme can avoid the Givens rotations as required in $[18,19]$. We first consider the QRD of the augmented channel matrix:

$$
\overline{\mathcal{H}}=\left[\begin{array}{c}
\mathcal{H} \\
\sigma_{v} \mathbf{I}_{B}
\end{array}\right]=\left[\begin{array}{l}
\mathcal{Q}_{F} \\
\mathcal{Q}_{B}
\end{array}\right] \mathcal{R}=\mathcal{Q R},
$$

where $\mathcal{H}=\mathbf{H F}_{Q R}, \mathcal{Q} \in C^{(M r+B) \times B}, \mathcal{R} \in C^{B \times B}, \mathcal{Q}_{F} \in C^{M r \times B}$ and $\mathcal{Q}_{B} \in C^{B \times B}$. The transmitted signals in MMSE detection can be estimated as

$$
\hat{\mathbf{x}}=\left(\mathcal{H}^{H} \mathcal{H}+\sigma_{v} \mathbf{I}_{B}\right)^{-1} \mathcal{H}^{H} \mathbf{y}=\overline{\mathcal{H}}^{\dagger}\left[\begin{array}{l}
\mathbf{y} \\
\mathbf{0}_{B}
\end{array}\right]=\mathcal{R}^{-1} \mathcal{Q}_{F}^{H} \mathbf{y}
$$

where $\overline{\mathcal{H}}^{\dagger}=\left(\overline{\mathcal{H}}^{H} \overline{\mathcal{H}}\right)^{-1} \overline{\mathcal{H}}^{H}$. Using (19), the interference cancellation in V-BLAST iterations indexed by $B, B-1, \ldots, 1$ yields $\mathcal{H}_{B}, \mathcal{H}_{B-1}, \ldots, \mathcal{H}_{1}$ with $\mathcal{H}_{B}=\mathcal{H}$ as described in the following.

Given the minimum-norm row $i$ with $i \leq B-m+1$ in the $m$ th iteration, $1 \leq m \leq B$, the ordered rows $i, i+1, i+2, \ldots, B-m, B-m+1$ of $\mathcal{R}_{B-m+1}^{-1}$ with $\mathcal{R}_{B}=\mathcal{R}$ is permuted into 
the new order $i+1, i+2, \ldots, B-m, B-m+1, i$. That is, we have $\mathbf{J}(B-m+1, i) \mathcal{R}_{B-m+1}^{-1}$ with $\mathbf{J}(B-m+1, i)$ being the permutation matrix with the minimum-norm row in the last row:

$$
\left[\begin{array}{c}
\overline{\mathcal{R}}_{B-m+1}^{-1} \\
\mathcal{R}_{B-m+1}^{-1}(i,:)
\end{array}\right]=\mathbf{J}(B-m+1, i) \mathcal{R}_{B-m+1}^{-1},
$$

where $\overline{\mathcal{R}}_{B-m+1}^{-1}=\left[\mathcal{R}_{B-m+1}^{-T}(1,:) \ldots \mathcal{R}_{B-m+1}^{-T}(i-1,:) \quad \mathcal{R}_{B-m+1}^{-T}(i+1,:) \ldots \mathcal{R}_{B-m+1}^{-T}\right.$ $(B-m+1,:)]^{T}, \mathcal{R}_{B-m+1}^{-T}(i,:)$ is the $i$ th row of $\mathcal{R}_{B-m+1}^{-T}$, and $\mathcal{R}_{B-m+1}^{-T}$ is the inverse of $\mathcal{R}_{B-m+1}^{T}$. In the $m$ th iteration, we have

$$
\begin{aligned}
& \mathbf{J}(B-m+1, i)\left(\mathcal{H}_{B-m+1}^{H} \mathcal{H}_{B-m+1}+\sigma_{v}^{2} \mathbf{I}_{B}\right)^{-1} \mathbf{J}(B-m+1, i) \mathcal{H}_{B-m+1}^{H} \\
& =\mathbf{J}(B-m+1, i) \mathcal{R}_{B-m+1}^{-1} \boldsymbol{\Sigma}_{B-m+1} \boldsymbol{\Sigma}_{B-m+1}^{H} \mathcal{R}_{B-m+1}^{-H} \mathbf{J}(B-m+1, i) \mathcal{H}_{B-m+1}^{H} \\
& =\left[\begin{array}{ll}
\mathcal{R}_{B-m}^{-1} & \mathbf{r}_{B-m+1} \\
\mathbf{0}_{B-m}^{T} & r_{B-m+1}
\end{array}\right]\left[\begin{array}{cc}
\mathcal{R}_{B-m}^{-1} & \mathbf{r}_{B-m+1} \\
\mathbf{0}_{B-m}^{T} & r_{B-m+1}
\end{array}\right]^{H} \mathcal{H}_{B-m+1}^{H} \\
& =\left[\begin{array}{cc}
\mathcal{R}_{B-m}^{-1} \mathcal{R}_{B-m}^{-H}+\mathbf{r}_{B-m+1} \mathbf{r}_{B-m+1}^{H} & \mathbf{r}_{B-m+1} r_{B-m+1} \\
r_{B-m+1} \mathbf{r}_{B-m+1}^{H} & r_{B-m+1} r_{B-m+1}
\end{array}\right] \mathcal{H}_{B-m+1}^{H},
\end{aligned}
$$

where $\Sigma_{B-m+1}$ represnets a sequence of Givens rotations for the upper triangular matrix $\mathcal{R}_{B-m}^{-1}$ [19]. The nulling vector is then given by

$$
\mathbf{g}_{B-m+1}=\left[\begin{array}{ll}
r_{B-m+1} \mathbf{r}_{B-m+1}^{H} & r_{B-m+1} r_{B-m+1}
\end{array}\right] \mathcal{H}_{B-m+1}^{H} .
$$

In "Appendix 2", it is shown that $r_{B-m+1}$ can be obtained by

$$
r_{B-m+1}=\left(\frac{\operatorname{det}\left(\mathcal{R}_{B-m+1}^{-1} \mathcal{R}_{B-m+1}^{-H}\right)}{\operatorname{det}\left(\overline{\mathcal{H}}_{B-m}^{-1} \overline{\mathcal{H}}_{B-m}^{-H}\right)}\right)^{1 / 2},
$$

without resorting to the Givens rotations. Given $r_{B-m+1}, \mathbf{r}_{B-m+1}$ can be obtained by

$$
\mathbf{r}_{B-m+1}=r_{B-m+1}^{-1} \mathbf{G}_{B-m+1}(1: B-m, B-m+1),
$$

where $\mathbf{G}_{B_{-m+1}}=\mathbf{J}(B-m+1, i)\left(\left(\mathbf{H}_{B_{-m+1}}\right)^{H} \mathbf{H}_{B_{-m+1}}+\sigma_{\mathrm{v}}^{2} \mathbf{I}_{B}\right)^{-1} \mathbf{J}(B-m+1, i)$. Using (22), the estimated signal in the $m$ th iteration is

$$
\tilde{x}_{B-m+1}=\operatorname{slice}\left(\mathbf{g}_{B-m+1} \mathbf{y}_{B-m+1}\right) .
$$

and interference cancellation due to $\tilde{x}_{B-m+1}$ is given by

$$
\mathbf{y}_{B-m}=\mathbf{y}_{B-m+1}-\mathbf{h}_{B-m+1} \tilde{x}_{B-m+1},
$$

Finally, the QRD of the augmented channel matrix is updated according to

$$
\left[\begin{array}{l}
\mathcal{H}_{B-m} \\
\sigma_{v} \mathbf{I}_{B-m}
\end{array}\right]=\left[\begin{array}{l}
\mathcal{Q}_{B-m, F} \\
\mathcal{Q}_{B-m, B}
\end{array}\right] \mathcal{R}_{B-m}=\mathcal{Q}_{B-m} \mathcal{R}_{B-m}
$$

By repeating the steps in (20)-(27), the proposed scheme exploiting the matrix determinant can implement the MMSE V-BLAST detection in an efficient manner and further improve the detection performance. In particular, the proposed scheme requires $\sum_{m=1}^{B-1} B-m+1$ multiplications in (22), which has a lower complexity than the scheme using the Givens rotations which requires $\frac{1}{2} \sum_{m=1}^{B-1}(B-m+1)^{3}$ multiplications [19]. 


\section{Performance Analysis}

In this section, the proposed QR-based selection criterion in multi-mode precoding for MUMIMO is analyzed in 4.1. Detection performance, and 4.2. Computational complexity.

\subsection{Detection Performance}

In this subsection, the detection performance achieved with the proposed QR-based selection criterion is analyzed. We first show that the QR-based selection criterion has a smaller distortion than the SV-based selection criterion [4] as follows.

Case 1: For $B<M_{t}$, when the number of substreams is given, the distortion between the optimal precoder and the selected precoder $[4,21]$ in the proposed QR-based selection scheme can be expressed as

$$
\begin{aligned}
\Delta & =E_{\mathbf{H}}\left\{\frac{\tilde{d}_{\min }^{2}(B, W)}{B}\left[\min _{1 \leq i \leq B} r_{i, i}^{2}\left(\mathbf{H E}_{M}\right)-\max _{\mathbf{F}_{M} \in \mathcal{F}_{\mathcal{M}}} \min _{1 \leq i \leq B} r_{i, i}^{2}\left(\mathbf{H F}_{M}\right)\right]\right\} \\
& \leq \frac{\tilde{d}_{\min }^{2}(B, W)}{B} E_{\mathbf{H}}\left[\bar{\lambda}^{2}(\mathbf{H})\left(1-\max _{\mathbf{F}_{M} \in \mathcal{F}_{\mathcal{M}}} \min _{1 \leq i \leq B} r_{i, i}^{2}\left(\mathbf{E}_{M}^{H} \mathbf{F}_{M}\right)\right)\right] \\
& \leq \frac{\tilde{d}_{\min }^{2}(B, W)}{B} E_{\mathbf{H}}\left[\bar{\lambda}^{2}(\mathbf{H})\left(1-\max _{\mathbf{F}_{M} \in \mathcal{F}_{\mathcal{M}}} \min _{1 \leq i \leq B} \lambda_{i}^{2}\left(\mathbf{E}_{M}^{H} \mathbf{F}_{M}\right)\right)\right]
\end{aligned}
$$

where the optimal precoder $\mathbf{E}_{M}$ satisfies $r_{i, i}\left(\mathbf{H E}_{M}\right)=\bar{\lambda}\left(\mathbf{H V} \mathbf{V}_{M}\right)$ with $\bar{\lambda}(\mathbf{H}):=\left(\prod_{i=1}^{B} \lambda_{i}\right.$ $\left.\left(\mathbf{H V} \mathbf{V}_{M}\right)\right)^{1 / B}, \mathbf{V}_{M}$ consists of the first $M$ columns of $\mathbf{V}^{H}$ with $\mathbf{H}=\mathbf{U} \boldsymbol{\Lambda} V^{H}$ being the SVD. Equation (28) shows that the QR-based selection criterion has a smaller distortion than SVbased selection criterion in [4] due to $\min _{1 \leq i \leq B} r_{i, i}^{2}\left(\mathbf{E}_{M}^{H} \mathbf{F}_{M}\right) \geq \min _{1 \leq i \leq B} \lambda_{i}^{2}\left(\mathbf{E}_{M}^{H} \mathbf{F}_{M}\right)$ [7].

Case 2: For $B=M_{t}$, the $i$ th precoder matrix is generated using the following Householder generating vector $\mathbf{u}_{i} \in C^{N t}$ [22]:

$$
\mathbf{F}_{M_{t}, i}=\mathbf{I}_{N_{t}}-\frac{2 \mathbf{u}_{i} \mathbf{u}_{i}^{H}}{\left\|\mathbf{u}_{i}\right\|^{2}} \quad \text { with } \quad \mathbf{F}_{M_{t}, i} \in \mathcal{F}_{M_{t}} .
$$

The distortion between the optimal precoder and selected precoder is given by (28). Thus, the efficacy of the proposed QR-based selection scheme can be confirmed and the associated distortion can be bounded as depicted in (28) in the construction of the codebook.

\subsection{Computational Complexity}

In this subsection, the computational complexity of the QR-based (with both full and partial search) [7] and SV-based multimode precoded systems [4] is investigated. The QR-based system requires the functions of the QR-based precoder selection combined with $\mathrm{QR}$ detection at the receiver. The SV-based system requires the functions of the SV-based precoder selection combined with MMSE detection at the receiver. The SV-based selection criteria to find the mode and precoder are similar to those found in (9)-(10). Table 3 presents the breakdowns of complexity in terms of complex multiplications associated with these precoding schemes. It can be noted in all schemes that the transmitter only requires multiplying the symbol vector by the precoding matrix determined at the receiver via the limited feedback channel, involving a complexity of $N_{1} M_{1}+N_{2} M_{2}+\cdots+N_{Q} M_{Q}$, as shown in (1). 
Table 3 Computational complexity for various MU-MIMO precoding systems

\begin{tabular}{|c|c|}
\hline $\begin{array}{l}\text { Selection criterion }+ \\
\text { detection }\end{array}$ & $+\underset{\text { Counts }}{\text { Complex }}$ \\
\hline
\end{tabular}

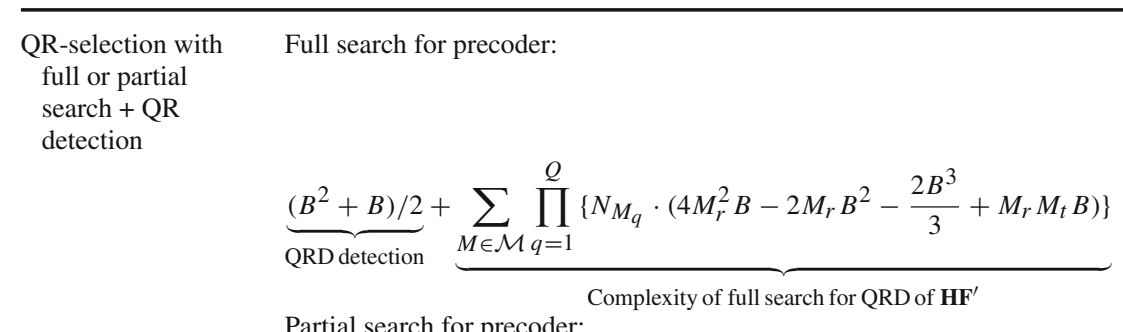

Partial search for precoder:

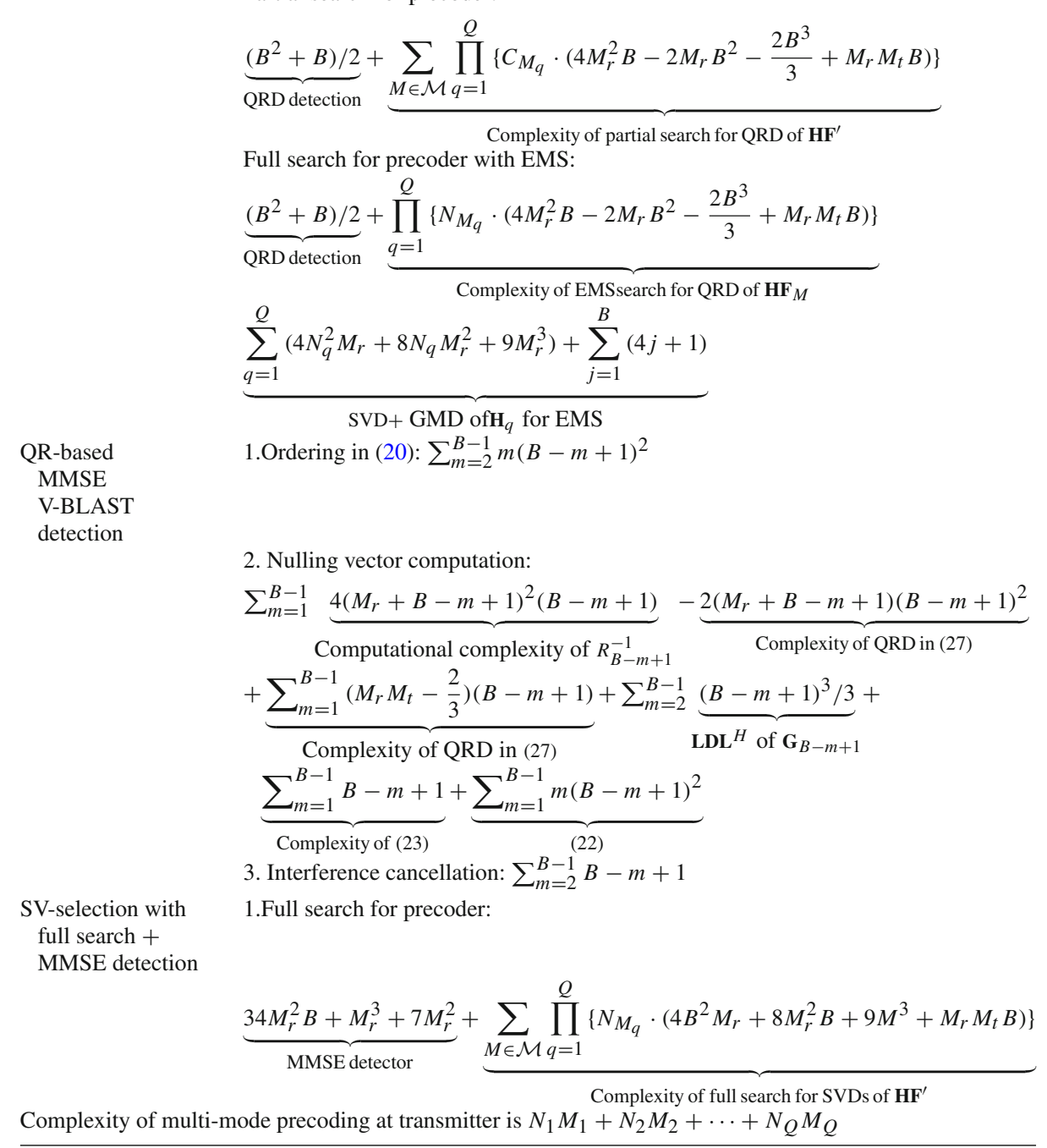


(i) With a full search in the QR-based system, the receiver involves precoder selection and the corresponding QR detection. The precoder selection involves a total of $\Sigma_{M \in \mathcal{M}}\left(N_{M 1} \cdot N_{M 2} \cdot \ldots N_{M Q}\right)$ QRDs of the effective channel matrix as shown in (3), each involving a complexity of $4 M_{r}^{2} B-2 M_{r} B^{2}-\frac{2 B^{3}}{3}$ [24] (for QRD of $\mathbf{H F}_{M}$ ) plus $M_{r} M_{t} B$ (for computing $\mathbf{H F}_{M}$ ). The detection part requires only a complexity of $\left(B^{2}+B\right) / 2$ since the same QRD used to identify the precoder is available for symbol detection.

(ii) For EMS, the receiver requires first determining a reference precoder by $\operatorname{SVD}\left(B<M_{t}\right)$ involving about a complexity of $4 B^{2} M_{r}+8 B M_{r}^{2}+9 M_{r}^{3}$ [23], or GMD $\left(B=M_{t}\right)$ involving about a complexity of $4 B^{2} M_{r}+8 B M_{r}^{2}+9 M_{r}^{3}+\sum_{j=1}^{B} 4(j+1)$. The Givens rotations require about a complexity of $\sum_{j=i}^{B} 4(j+1)$ for the worse case [19].

(iii) For tree search, the precoder selection requires a total of $\Sigma_{M \in \mathcal{M}}\left(C_{M 1} \cdot C_{M 2} \cdot \ldots C_{M Q}\right)$ QRDs of the effective channel matrix as in (3), each having a complexity of $4 M_{r}^{2} B-$ $2 M_{r} B^{2}-\frac{2 B^{3}}{3}$ (for QRD of $\mathbf{H} \mathbf{F}_{M}$ ) plus $M_{r} M_{t} B$ (for computing $\mathbf{H F}_{M}$ ). The complexity of a binary search is about $\log _{2} N M_{q}$ for the worse case. The complexity for precoders converted to a binary search tree can be neglected since precoders of the codebook are known before transmission.

(iv) For QR-based MMSE V-BLAST detection in Table 3, a complexity of $\sum_{m=2}^{B-1} m(B-$ $m+1)^{2}$ for ordering, a complexity of $4\left(M_{r}+B\right)^{2} B-2\left(M_{r}+B\right) B^{2}-2 B^{3} / 3+M_{r} M_{t} B$ for the QRD of $\bar{H}$ and a complexity of $B^{3} / 3+B$ for computing the inverse term in (19) by using $\mathrm{LDL}^{H}$ are required, respectively.

(v) For SV-based systems, the receiver needs to do precoder selection and the corresponding MMSE detection. The precoder selection involves a total of $\Sigma_{M \in \mathcal{M}}\left(N_{M 1} \cdot N_{M 2}\right.$. $\left.\ldots N_{M Q}\right)$ SVDs of the effective channel matrix, each having a complexity of $4 B^{2} M_{r}+$ $8 M_{r}^{2} B+9 B^{3}$ [24] (for SVD of $\mathbf{H F}_{M}$ ) plus $M_{r} M_{t} B$ (for computing $\mathbf{H F}_{M}$ ). The MMSE detection needs a complexity of $34 M_{r}^{2} B+M_{r}^{3}+7 M_{r}^{2}$.

As anticipated, Table 3 shows that with the reduction of QRD computations, the EMS and tree search schemes effectively reduce the computational complexity substantially.

\section{Simulation Results}

This section uses several numerical examples to demonstrate the bit error rate (BER) performance of the proposed schemes for multi-mode precoding in an uplink MU-MIMO system. The considered codebooks are obtained from [25] (for $B<M_{t}$ ) and [22] (for $B=M_{t}$ ), respectively. For a fair comparison, all precoders are properly normalized in accordance with $\mathcal{F}_{1}, \mathcal{F}_{2} / \sqrt{2}$ and $\mathcal{F}_{4} / 2$ for $M_{q}=1, M_{q}=2$ and $M_{q}=4$, respectively; so the total transmit power is maintained equal for all transmission modes. This multi-mode precoding system with feedback informs the transmitter of the determined precoder assuming the perfect CSI at the receiver. Assuming a Ricean channel, $\mathbf{H}_{\mathrm{sp}}$ indicates the specular component that is spatially deterministic from one antenna to another, and $\mathbf{H}_{\mathrm{sc}}$ indicates the scattered component that varies randomly from one antenna to another (Rayleigh-distribution). The channel response is thus given by [26]

$$
\mathbf{H}=\sqrt{\frac{\kappa}{-\kappa+1}} \mathbf{H}_{\mathrm{sp}}+\sqrt{\frac{1}{\kappa+1}} \mathbf{H}_{\mathrm{sc}},
$$

where the Ricean factor $\kappa$ denotes as the deterministic-to-scattered power ratio; the channel is rich-scattered as $\kappa \rightarrow 0$ and the channel is strongly correlated as $\kappa \rightarrow \infty$. The examples 

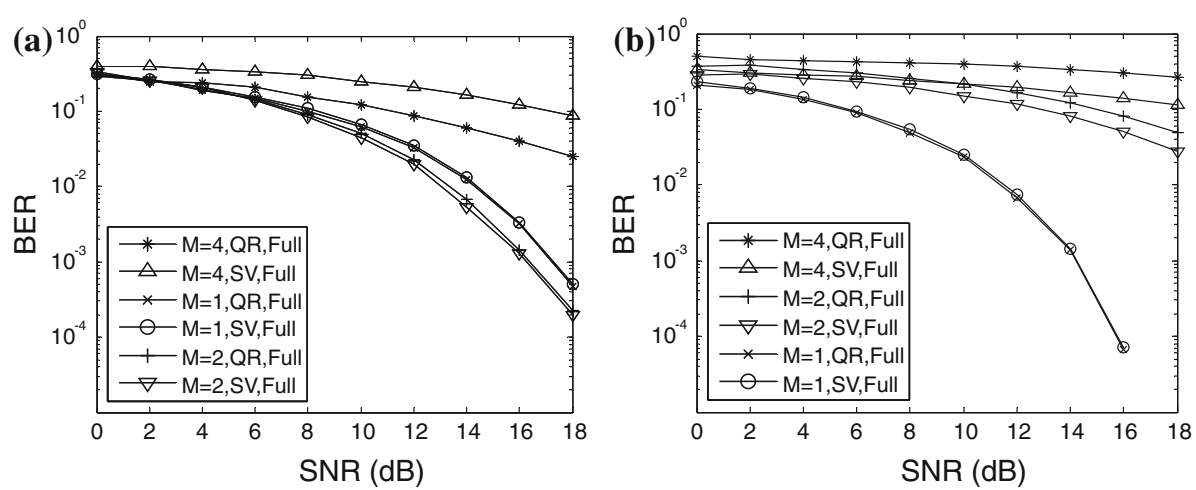

Fig. 2 BER versus SNR for an SU single mode precoded MIMO system $(\mathcal{M}=\{1\}$, $\{2\}$ and $\{4\})$ with $M_{t}=M_{r}=4, N_{1}=N_{2}=64, N_{4}=16$ and $W=4$ bits/symbol. a $\kappa=0 ; \mathbf{b} \kappa=10$

$\kappa=0$ and $\kappa=10$, respectively, correspond to the channels with independent fading and being almost light-of-sight. In the following, we evaluate the proposed schemes in 5.1 Detection performance of SU-MIMO precoding with EMS and 5.2 Detection performance of MU-MIMO precoding.

\subsection{Detection Performance of SU-MIMO Precoding with EMS}

In this set of simulations, the effectiveness of mode selection is investigated. SU-MIMO precoding with $M_{t}=M_{r}=4, Q=1$ and $W=4$ bits/symbol is considered, and both the single mode precoder and multi-mode precoder with EMS are assessed.

Case 1: we consider single mode precoding with $\mathcal{M}=\{1\},\{2\}$ and $\{4\}$, respectively, and demonstrate the performance of the QR-based selection criteria via (9) (QR, full) in conjunction with $\mathrm{QR}$ detection, and via SV-based selection criteria $(\mathrm{SV}$, full) in conjunction with MSE detection at [4] for $\kappa=0$ and $\kappa=10$. With a well-conditioned channel by $\kappa=0$, Fig. 2a shows that $M=2$ provides the best detection performance compared to others. This is because the system is able to support two effective spatial channels with a smaller singular value spread. On the other hand, with an ill-conditioned channel by $\kappa=10$, Fig. 2 b shows that $M=2$ provides worse detection performance than $M=1$ leading to the opposite trend to Fig. 2a. For the correlated channel with $\kappa=10, M=1$ (beamforming) would naturally be the best strategy to adopt since the SU-MIMO system is only able to support one effective spatial channel with a large singular value spread. In both cases, $M=4$ gives the worst performance because the system cannot reliably support four spatial channels. It is noteworthy that for the optimal mode, the proposed QR-based selection criteria with QR detection results in a similar detection performance compared to the more complicated SV-based selection criterion with MMSE detection, confirming the effective bound for mode/precoder selection described in Sect. 4.1.

Case 2: we consider multi-mode precoding with $\mathcal{M}=\{1,2,4\}$, and evaluate the performance of the QR-based selection criteria via (9)-(10) (MM, QR, full) and EMS in (12)-(13) (MM, QR, EMS) in conjunction with QR detection, and SV-based selection criterion (MM, $\mathrm{SV}$, full) in conjunction with MMSE detection [4]. With $\kappa=0$, Fig. 3a shows that $M=2$ is selected for this channel condition confirming the result in Fig. 2a. With $\kappa=10$, Fig. $3 \mathrm{~b}$ shows that $M=1$ (i.e., beamforming) is selected for this correlated channel confirming the result in Fig. 2b. In both cases, the proposed EMS scheme has nearly the same detection per- 

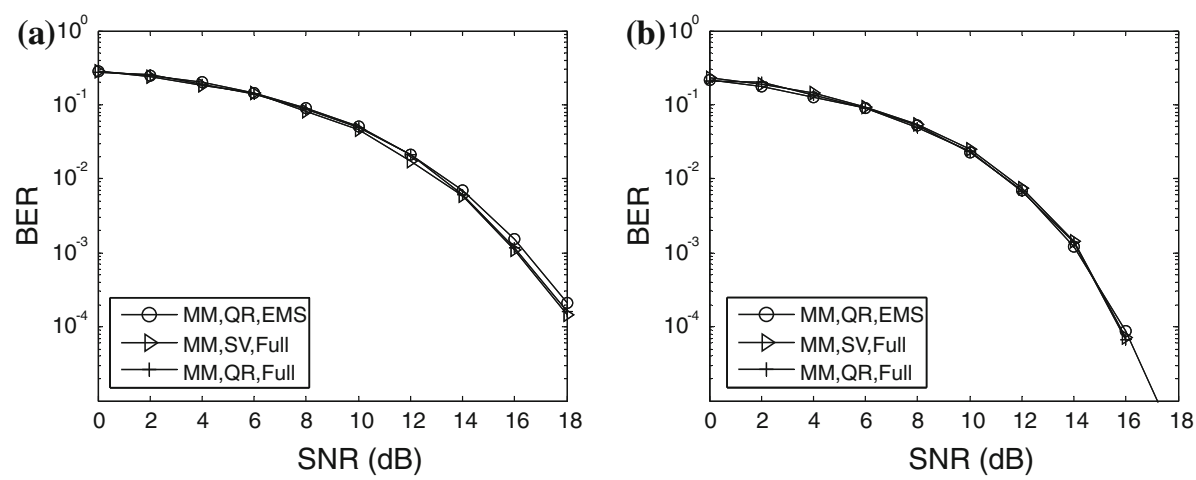

Fig. 3 BER versus SNR for an SU multi-mode precoded MIMO system $(\mathcal{M}=\{1,2,4\})$ with $M_{t}=M_{r}=$ $4, N_{1}=N_{2}=64, N_{4}=16$ and $W=4$ bits/symbol. $\mathbf{a} \kappa=0 ; \mathbf{b} \kappa=10$

Table 4 Complex multiplication counts of QR- and SV-based SU-MIMO precoded systems

\begin{tabular}{llll}
\hline Selection criteria & $(\mathrm{MM}, \mathrm{QR}$, Full $)$ & $(\mathrm{MM}, \mathrm{QR}$, EMS $)$ & $(\mathrm{MM}, \mathrm{SV}$, Full $)$ \\
\hline Multiplications & 14,803 & 8,283 & 61,744 \\
\hline
\end{tabular}

formance compared to the full search schemes, confirming the effectiveness of the reduced complexity mode selection scheme described in Sect. 3.1.

Table 4 compares the counts of complex multiplications per execution of the proposed (full and EMS) and SV-based precoded systems with the previous simulation setting. As observed, the QR-based scheme employing EMS can reduce the overall computational complexity (precoder selection plus detection) by about 45 and $87 \%$ compared to QR-based full and SVbased full schemes, respectively. This reduction in complexity confirms the previous results summarized in Table 3.

\subsection{Detection Performance of MU-MIMO Precoding}

In this set of simulations, MU-MIMO multi-mode precoding with $M_{t}=M_{r}=8, N_{q}=W_{q}=$ 4, $Q=2, M_{q} \in\{1,2,4\}$ and $W=8$ bits/symbol is considered, and both schemes with full precoder search $\left(N_{M 1}=N_{M 2}=64\right.$ and $\left.N_{M 4}=16\right)$ and tree search $\left(C_{M 1}=C_{M 2}=3\right.$ and $\left.C_{M 4}=2\right)$ are assessed.

Case 1: we evaluate the multi-mode detection performance of the QR-based selection criteria via (9)-(10) (MM, QR, Full) and via the tree search summarized in Table 2 (MM, $\mathrm{QR}$, Tree) in conjunction with QR detection, and SV-based selection criterion (MM, SV, Full) in conjunction with MMSE detection [4]. The proposed EMS is also incorporated to give the result (MM, QR, EMS, Tree). With a well-conditioned channel by $\kappa=0$, Fig. 4a shows that the QR-based selection criterion (MM, QR, Full) has a better detection performance than SV-based selection criterion due to a smaller distortion bound as described in Sect. 4.1. With the large singular value spread by $\kappa=10$, Fig. 4b shows that the proposed QR-based scheme has a slightly worse detection performance due to the error propagation with a poor detection ordering. To remedy this, the effect of column-ordering will be demonstrated in the next set of simulations.

Figure 4 also shows that the proposed QR-based selection criterion via full mode selection and tree search (MM, QR, Tree) and EMS with tree search (MM, QR, EMS, Tree) maintains 

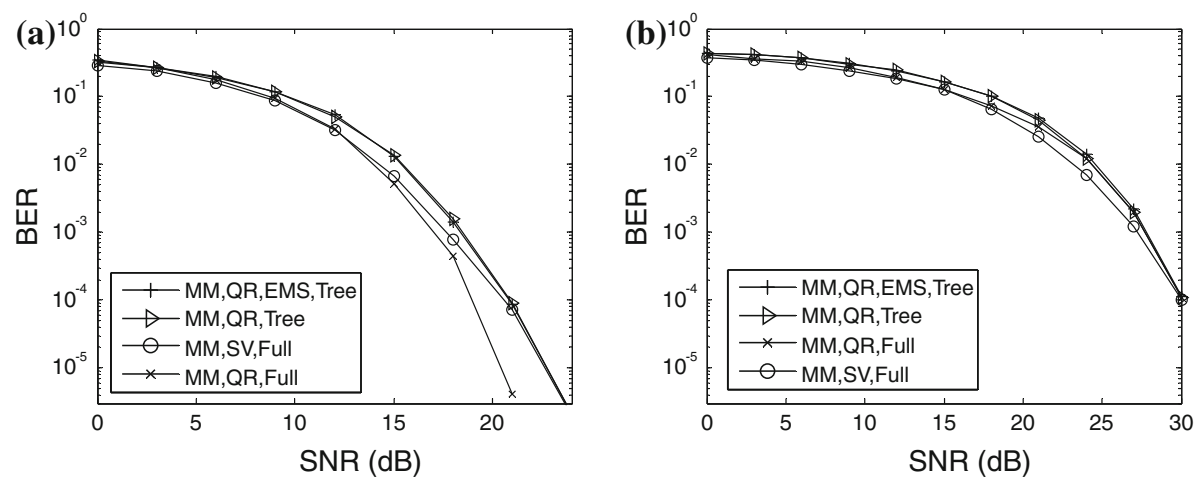

Fig. 4 BER versus SNR for an MU multi-mode precoded MIMO system with $M_{t}=M_{r}=8, Q=2$ and $W_{q}=N_{q}=4$ bits/symbol. $\mathbf{a} \kappa=0 ; \mathbf{b} \kappa=10$

Table 5 Complex multiplication counts of QR- and SV-based MU-MIMO precoded systems

\begin{tabular}{lllll}
\hline Selection criterion & (MM, QR, Full) & (MM, QR, Tree) & (MM, QR, EMS, Tree) & (MM, SV, Full) \\
\hline Multiplications & $17,978,118$ & 61,058 & 23,302 & $64,089,836$ \\
\hline
\end{tabular}

a reliable detection performance compared to the full search scheme, though a slight drop is observed.

Table 5 compares the complex multiplication counts per execution of the proposed (full, tree search and EMS) and SV-based precoded systems in the previous simulation setting. As observed, the QR-based scheme employing EMS and tree search (MM, QR, EMS, Tree) can reduce the overall computational complexity (precoder selection plus detection) by about 99.9 and $99.9991 \%$ compared to QR-based and SV-based full schemes, respectively. This reduction in complexity confirms the previous results summarized in Table 3.

Case 2: we evaluate the multi-mode detection performance of the QR-based selection criteria via (9)-(10) (MM, QR, Full) combined with QR detection, the proposed QR-based MMSE V-BLAST detection (MM, QR, Full, Ordering) in (18)-(27), and SV-based selection criterion (MM, SV, Full) combined with MMSE detection [4]. In MU-MIMO precoding, detection ordering is essential for an ill-conditioned channel because the total number of data streams is at least two, as mentioned in Lemma 1. In this case, the effect of error propagation will degrade the detection performance as illustrated in Fig. 4b. With the proposed QRbased MMSE V-BLAST detection incorporated, the results in Fig. 5 show that the BER performance of the QR-based precoder is effectively improved. In particular, Fig. 5b shows that the proposed QR-based MMSE V-BLAST detection overcomes the error propagation problem in ill-conditioned channels.

\section{Conclusion}

In this paper, we propose efficient QR-based schemes for multi-user multi-mode precoding in limited feedback MIMO systems. The MU-MIMO precoding is developed which takes advantage of the overall precoder design among users in conjunction with QR detection. EMS and tree search strategies are also suggested to further reduce the complexity of the precoder selection procedure. To improve the detection performance effectively in correlated channels, 

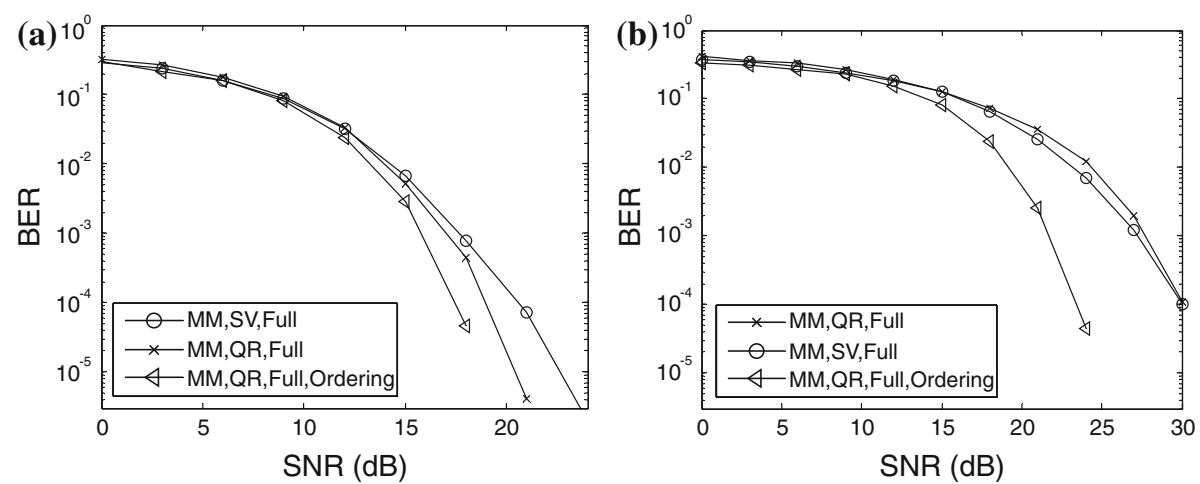

Fig. 5 BER versus SNR for an MU multi-mode precoded MIMO system with $M_{t}=M_{r}=8, Q=2$ and $W_{q}=N_{q}=4$ bits/symbol. a $\kappa=0 ; \mathbf{b} \kappa=10$

an efficient QR-based MMSE V-BLAST detection scheme is proposed. Performance analysis and simulation results confirm that the proposed efficient QR-based precoding and detection schemes can effectively optimize the error probability with a very lower computational complexity. The proposed precoded system is a promising solution for practical MIMO wireless communication systems for which implementation complexity at the mobile station is of major concerns.

Acknowledgments This work was supported by the National Science Council of Taiwan under Contract NSC 99-2221-E-009-098-MY3, by the Ministry of Education of Taiwan under MoE ATU Program, and by MediaTek, Inc. under the project "Essential Technologies for Advanced Wireless Communication System.

Open Access This article is distributed under the terms of the Creative Commons Attribution License which permits any use, distribution, and reproduction in any medium, provided the original author(s) and the source are credited.

\section{Appendix 1: Proof of Theorem 1}

Proof With $\mathbf{E}_{M q}=\mathbf{V}_{M q}$ for $M_{q}<N_{q}$, the free distance between two symbols for the $\left(M_{q}\right)^{t h}$ mode is given by

$$
\begin{aligned}
d_{\text {free }}\left(\mathbf{H}_{q} \mathbf{E}_{M_{q}}\right) & =\min _{\mathbf{s}_{q}, \mathbf{c}_{q} \in \chi^{M_{q}}, \mathbf{s}_{q} \neq \mathbf{c}_{q}}\left\|\mathbf{H}_{q} \mathbf{E}_{M_{q}}\left(\mathbf{s}_{q}-\mathbf{c}_{q}\right)\right\|=\min _{\mathbf{s}_{q}, \mathbf{c}_{q} \in \chi^{M_{q}, \mathbf{s}_{q} \neq \mathbf{c}_{q}}}\left\|\boldsymbol{\Lambda}_{M_{q}}\left(\mathbf{s}_{q}-\mathbf{c}_{q}\right)\right\| \\
& =\min _{1 \leq i \leq M_{q}}\left|r_{i, i}^{q}\left(\mathbf{H}_{q} \mathbf{E}_{M_{q}}\right)\right| \cdot d_{\min }\left(M_{q}, W_{q}\right)
\end{aligned}
$$

where (31) holds due to property 1. With $\mathbf{E}_{M q}=\mathbf{P}_{q}$ for $M_{q}=N_{q}$, the free distance between two symbols for the $\left(M_{q}\right)$ th mode is given by

$$
d_{\text {free }}\left(\mathbf{H}_{q} \mathbf{E}_{M_{q}}\right)=\min _{\mathbf{s}_{q}, \mathbf{c}_{q} \in \chi^{M_{q}}, \mathbf{s}_{q} \neq \mathbf{c}_{q}}\left\|\mathbf{H}_{q} \mathbf{E}_{M_{q}}\left(\mathbf{s}_{q}-\mathbf{c}_{q}\right)\right\|=r \cdot d_{\min }\left(M_{q}, W_{q}\right),
$$

where (32) holds since $r_{1,1}=r_{2,2}=\cdots=r_{M q, M q}=r$ according to the property of GMD of [15] and the proof is thus complete. 


\section{Appendix 2: Proof of (22)}

Proof By using Givens rotations in [19], $\mathbf{J}(B-m+1, i) \mathcal{R}_{B-m+1}^{-1}$ are rotated into a triangular matrix with a rotated sequence $\Sigma_{B-m+1}=\mathbf{G}(B-m+1, i) \mathbf{G}(B-m+1, i+1) \ldots \mathbf{G}(B-m+$ $1, B-m)$, where the Givens rotation $\mathbf{G}(B-m+1, i)$ is used to eliminate the $(B-m+1, i)$ th non-zero entry of $\mathcal{R}_{B-m+1}^{-1}$. That is, the permutation and the Givens rotations for $\mathcal{R}_{B-m}^{-1}$ can be

$$
\begin{aligned}
\mathbf{J}(B & -m+1, i) \mathcal{R}_{B-m+1}^{-1} \underbrace{\mathbf{G}(B-m+1, i) \mathbf{G}(B-m+1, i+1) \cdots \mathbf{G}(B-m+1, B-m)}_{=\mathbf{\Sigma}_{B-m+1}} \\
& =\left[\begin{array}{ll}
\mathcal{R}_{B-m}^{-1} & \mathbf{r}_{B-m} \\
\mathbf{0}_{B-m}^{T} & r_{B-m+1}
\end{array}\right],
\end{aligned}
$$

where $\mathbf{r}_{B-m} \in C^{B-m}$ and $\mathcal{R}_{B-m}^{-1}$ is determined for the next iteration [19]. Without Givens rotations, $r_{B-m+1}$ also can be computed by using the matrix determinant on (33) as

$$
r_{B-m+1}=\left(\frac{\operatorname{det}\left(\mathcal{R}_{B-m+1}^{-1} \mathcal{R}_{B-m+1}^{-H}\right)}{\operatorname{det}\left(\mathcal{R}_{B-m}^{-1} \mathcal{R}_{B-m}^{-H}\right)}\right)^{1 / 2},
$$

where (34) holds due to $\operatorname{det}\left(\mathcal{R}_{B-m}^{-1} \mathcal{R}_{B-m}^{-H}\right)=\operatorname{det}\left(\overline{\mathcal{H}}_{B-m}^{-1} \overline{\mathcal{H}}_{B-m}^{-H}\right), \operatorname{det}(\mathbf{J}(B-m+1, i))=1$ and $\operatorname{det}\left(\boldsymbol{\Sigma}_{B-m+1}\right)=1$. Thus, proof is complete.

\section{References}

1. Lin, Z. H., Xiao, P., \& Vucetic, B. (2010). Analysis of receiver algorithms for LTE SC-FDMA based uplink MIMO systems. IEEE Transactions on Wireless Communication, 9(1), 60-65.

2. Sawahashi, M., Kishiyama, Y., Morimoto, A., Nishikawa, D., \& Tanno, M. (2010). Coordinated multipoint transmission/reception techniques for LTE-advanced. IEEE Transactions on Wirelsee Communication Magazine, 17(3), 26-34.

3. Ho, W. W. L., Quek, T. Q. S., Sun, S., \& Heath, R. W. (2011). Decentrailized precoding for multicell MIMO downlink. IEEE Transactions on Wireless Communication, 10(6), 1798-1809.

4. Love, D. J., \& Heath, R. W, Jr. (2005). Multimode precoding for MIMO wireless systems. IEEE Transactions on Signal Processing, 53(10), 3674-3687.

5. Zhong, W., Xu, Y., Tao, M., \& Cai, Y. (2010). Game theoretic multimode precoding strategy selection for MIMO multiple access channels. IEEE Transactions on Signal Processing Letter, 17(6), 563-566.

6. Lau, V. K. N., Liu, Y., \& Chen, T.-A. (2004). On the design of MIMO block-fading channels with feedback-link capacity constraint. IEEE Transactions on Communication, 52(6), 62-70.

7. Pan, C. H., \& Lee, T. S. A low complexity QR-based selection criterion for MIMO precoding. In Proceedings of the APSIPA ASC 2011, Xi'an. Oct: China, 2011.

8. Kahled, N., Mondal, B., Leus, G., Heath, R. H, Jr, \& Petré, F. (2007). Interpolation-based multi-mode precoding for MIMO-OFDM systems with limited feedback. IEEE Transactions on Wireless Communication, 6(3), 1003-1013.

9. Kim, J. K., Pun, M. O., \& Iltis, R. A. (2010). QRD-based precoded MIMO-OFDM systems with reduced feedback. IEEE Transactions on Communication, 58(2), 394-398.

10. Gesbert, D., Kountouris, M., Heath, R. W, Jr, Chae, C.-B., \& Salzer, T. (2007). From single user to multiuser communications: shifting the MIMO paradigm. IEEE Transactions on Signal Processing Magazine, 24(5), 36-46.

11. Huang, K., \& Zhang, R. (2011). Cooperative feedback for multi-antenna cognitive radio networks. IEEE Transactions on Signal Processing, 59, 747-758.

12. Tenenbaum, A. J., \& Adve, R. S. (2009). Linear processing and sum throughput in the multiuser MIMO antenna system. IEEE Transactions on Wireless Communication, 8(5), 2652-2661.

13. Pan, C. H., Lee, T. S., \& Li, Y. M. (September 2007). An efficient near-ML algorithm with SQRD for wireless MIMO communications in metro transportation systems. In Proceedings of the IEEE ITSC (pp. 603-606). Seattle, WA, USA. 
14. Zhang, J. K., Kavcic, A., \& Wong, K. M. (2005). Equal-diagonal QR decomposition and its application to precoder design for successive-cancellation detection. IEEE Transactions on Information Theory, 51(1), 154-172.

15. Jiang, Y., Li, J., \& Hager, W. (2005). Joint transceiver design for MIMO communications using geometric mean decomposition. IEEE Transactions on Signal Processing, 53(10), 3791-3803.

16. Santipach, W. (2008). Tree-structured random vector quantization for beamforming in a multiantenna channel. In Proceedings of the ECTI-CON, (Vol. pp. 325-328.) 1, Krabi, Thailand. May 2008

17. Guo, Z., \& Nilsson, P. (2006). Algorithm and implementation of the K-Best sphere decoding for MIMO detection. IEEE Journal on Selected Areas in Communication, 24(3), 491-503.

18. Hassibi, B. (2000). An efficient square-root algorithm for BLAST. In Proceedings of the International Conference Accoustic Speech Signal Processing (ICASSP) (pp. 737-740).

19. Liu, T.-H. (2006). Efficient and stable MMSE detection of the V-BLAST system. In Proceedings of the IEEE global telecommunication Conference (GLOBECOM) (pp. 1-5). San Franciso, USA, Nov 2006.

20. Chen, X., Hu, H., Wang, H., Chen, H.-H., \& Guizani, M. (2008). User pairing transmission scheme in uplink coordinated multi-point reception. IEEE Transactions on Wireless Communication, 7(7), 2425-2429.

21. Love, D. J., Heath, R. W, Jr., \& Strohmer, T. (2003). Grassmannian beamforming for multiple-input multiple-output wireless systems. IEEE Transactions on Information Theory, 49(10), 2735-2747.

22. Khan, F. (2009). LTE for $4 G$ mobile broadband-air interface technologies and performance. New York: Cambridge University Press.

23. Golub, G. H., \& Van Loan, C. F. (1996). Matrix computations (3rd ed.). Baltimore: The Johns Hopkings University Press.

24. Toshiaki, K. (2009). Low-complexity systolic V-BLAST architecture. IEEE Transactions on Wireless communication, 8(5), 2172-2176.

25. Love, D. Grassmanian subspace packing [Online]. Available: http://dynamo.ecn.purdue.edu/ djlove/ grass.html.

26. Farrokhi, F. R., Soschini, G. J., Lozano, A., \& Valenzuela, R. A. (2001). Link-optimal space-time processing with multiple transmit and receiver antennas. IEEE Communication Letter, 5(3), 85-87.

\section{Author Biographies}

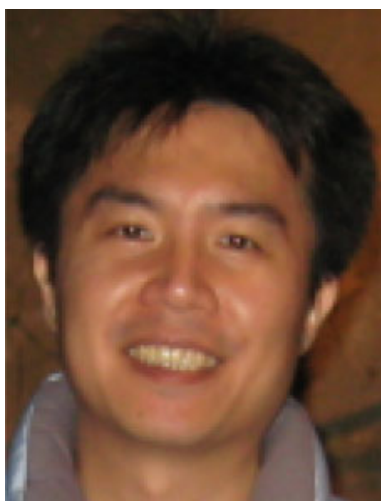

Chien-Hung Pan received the B.S. degree in Department of Electronic and Computer Engineering from National Taiwan University of Science and Technology, Taipei, Taiwan, and the M.S. and Ph.D. degrees in Institute of Communications Engineering from National Chiao Tung University, Hsinchu, Taiwan. His current research focuses on multimode transmission, precoder selection and interference cancellation for wireless MIMO communication. 


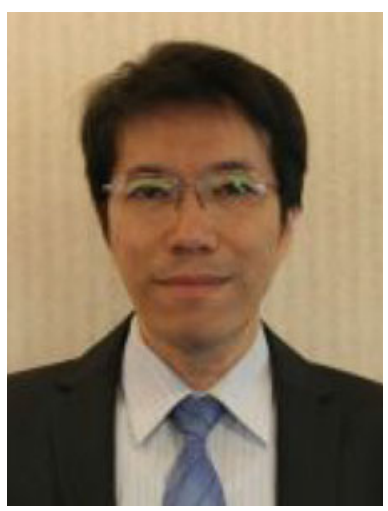

Ta-Sung Lee received the B.S. degree from National Taiwan University in 1983, the M.S. degree from University of Wisconsin, Madison, in 1987, and the Ph.D. degree from Purdue University, W. Lafayette, IN, in 1989, all in electrical engineering. In 1990, he joined the Faculty of National Chiao Tung University (NCTU), Hsinchu, Taiwan, where he holds a position as Professor of Department of Electrical Engineering. From 2005 to 2007, he was Chairman of Department of Communication Engineering, and from 2007 to 2008, he was Dean of Student Affairs of NCTU. From 2008 to 2010, he was Commissioner of National Communications Commission (NCC) and responsible for the strategic planning, policy making and regulation of technologies and resources for the entire sector of telecommunications and broadcasting services in Taiwan. He was Vice Chairman and Chairman of IEEE Communications Society Taipei Chapter from 2005 to 2008, and a Board Member of IEEE Taipei Section from 2007 to 2010. He is currently IEEE Signal Processing Society Regional Director-at-Large for Region 10. Dr. Lee has been actively involved in research and development in signal processing and system design for wireless communications. He has won several awards for his research, engineering and education contributions; these include National Science Council (NSC) Research Award, 1999 Young Electrical Engineer Award of the Chinese Institute of Electrical Engineering (CIEE), 2011 Distinguished Electrical Engineering Professor Award of CIEE, NCTU Distinguished Scholar Award, and NCTU Teaching Award. 